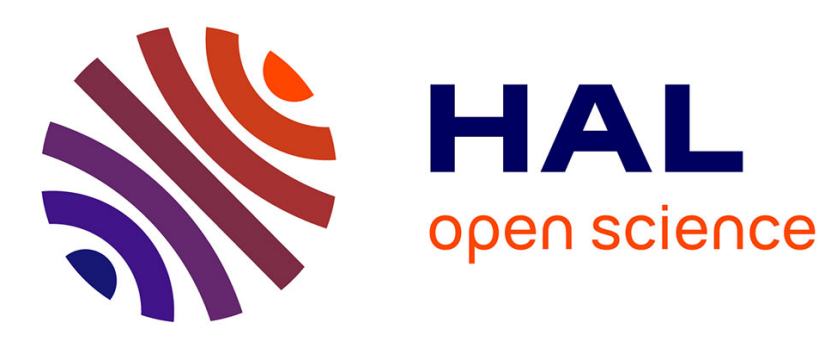

\title{
Observation of the strain induced magnetic phase segregation in manganite thin films
}

Lorena Marín, Luis A. Rodríguez, César Magén, Etienne Snoeck, Rémi Arras, Irene Lucas, Luis Morellón, Pedro A. Algarabel, José M. de Teresa, M.

Ricardo Ibarra

\section{To cite this version:}

Lorena Marín, Luis A. Rodríguez, César Magén, Etienne Snoeck, Rémi Arras, et al.. Observation of the strain induced magnetic phase segregation in manganite thin films. Nano Letters, 2015, 15 (1), pp.492-497. 10.1021/nl503834b . hal-01718310

\section{HAL Id: hal-01718310 https://hal.science/hal-01718310}

Submitted on 15 Mar 2018

HAL is a multi-disciplinary open access archive for the deposit and dissemination of scientific research documents, whether they are published or not. The documents may come from teaching and research institutions in France or abroad, or from public or private research centers.
L'archive ouverte pluridisciplinaire HAL, est destinée au dépôt et à la diffusion de documents scientifiques de niveau recherche, publiés ou non, émanant des établissements d'enseignement et de recherche français ou étrangers, des laboratoires publics ou privés. 
Observation of the strain induced magnetic phase segregation in manganite thin films

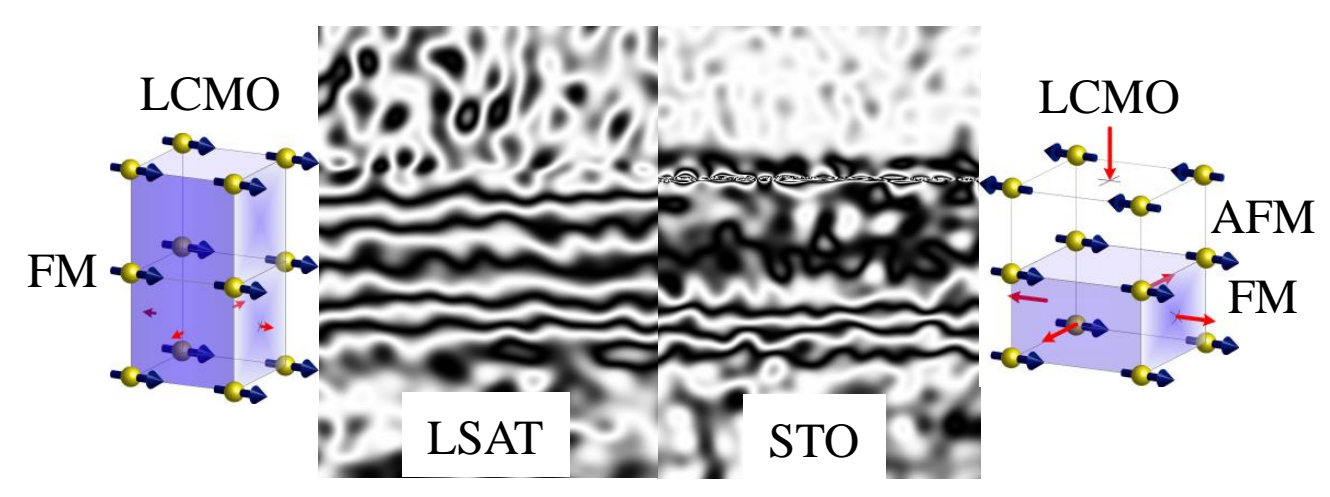




\title{
Observation of the Strain Induced Magnetic Phase Segregation in Manganite Thin Films
}

\author{
Lorena Marín $^{1,2,3, \dagger}$, Luis A. Rodríguez ${ }^{2,4,5,6, \dagger}$, César Magén ${ }^{2,4,5,6, *}$, Etienne Snoeck ${ }^{5,7}$, \\ Rémi Arras ${ }^{5,7}$, Irene Lucas ${ }^{1,6}$, Luis Morellón ${ }^{1,7}$, Pedro A. Algarabel ${ }^{2,3}$, José M. De \\ Teresa ${ }^{2,3,4,5}$ and M. Ricardo Ibarra ${ }^{2,4,5}$
}

1. Instituto de Nanociencia de Aragón (INA), Universidad de Zaragoza, 50018 Zaragoza, Spain.

2. Departamento de Física de la Materia Condensada, Universidad de Zaragoza, 50009 Zaragoza, Spain.

3. Instituto de Ciencia de Materiales de Aragón (ICMA), Universidad de Zaragoza, 50018 Zaragoza, Spain

4. Laboratorio de Microscopias Avanzadas (LMA), Instituto de Nanociencia de Aragón (INA), Universidad de Zaragoza, 50018 Zaragoza, Spain.

5. Transpyrenean Associated Laboratory for Electron Microscopy (TALEM), CEMESINA, CNRS-Universidad de Zaragoza, Toulouse, France.

6. Fundación ARAID, 50018 Zaragoza, Spain.

7. CEMES-CNRS 29, rue Jeanne Marvig, B.P. 94347 F-31055, Toulouse Cedex, France.

$\dagger$ These authors have contributed equally to this work:

Lorena Marín and Luis A. Rodríguez

*Author to whom the correspondence should be addressed:

César Magén (ㅆagend@ unizar.es), Tel: +34 876 555369, Fax: +34 976762776. 


\section{ABSTRACT}

Epitaxial strain alters the physical properties of thin films grown on single crystal substrates. Thin film oxides are particularly apt for strain engineering new functionalities in ferroic materials. In the case of $\mathrm{La}_{2 / 3} \mathrm{Ca}_{1 / 3} \mathrm{MnO}_{3}$ (LCMO) thin films, here we show the first experimental images obtained by electron holography demonstrating that epitaxial strain induces the segregation of a flat and uniform nonferromagnetic layer with antiferromagnetic (AFM) character at the top surface of a ferromagnetic (FM) layer, the whole film being chemical and structurally homogeneous at room temperature. For different substrates and growth conditions the tetragonality of LCMO at room temperature, defined as $\tau=|c-a| / a$, is the driving force for a phase coexistence above an approximate critical value of $\tau_{C} \sim 0.024$. Theoretical calculations prove that the increased tetragonality changes the energy balance of the FM and AFM ground states in strained LCMO, enabling the formation of magnetically inhomogeneous states. This work gives the key evidence that opens a new route to synthesize strain-induced exchanged-biased FM-AFM bilayers in single thin films, which could serve as building blocks of future spintronic devices.

\section{KEYWORDS:}

Strain engineering, manganites, epitaxial thin films, electron holography, phase segregation, exchange bias. 


\section{MAIN TEXT}

The progress of semiconductor technology in the last decades has been based on continuous increasing of areal density of transistors validating the Moore's law. However, scientific community is continuously investigating alternatives to anticipate the time when miniaturization of nanoelectronic devices with current technology reaches its physical limits. Engineering smart multifunctional materials is one of these possible alternatives, and complex oxides are the model materials for this paradigm. Subtle changes of the chemical composition, symmetry or electronic structure induce multiple functionalities such as multiferroism ${ }^{1}$ or superconductivity ${ }^{2}$. Manganites are one of the archetypes in the field of functional complex oxides, as they combine numerous exotic properties interplayed, such as colossal magnetoresistance (CMR) and phase coexistence at a metal-insulator transition, half metallicity, orbital and charge ordering, etc., which make them appealing candidates for fundamental studies as well as for spintronic applications. Furthermore, their perovskite crystal structure allows the growth of epitaxial thin films and the fabrication of high quality heterostructured devices ${ }^{3}$.

The implementation of devices often involves the epitaxial growth of thin films of these functional oxides. This requires a support (substrate) that imposes certain constraints related to the symmetry and the lattice parameter of the material to be grown. However, the substrate constraints can be used to induce new properties and the type of substrate can be smartly chosen as well as the growth conditions so the substrate-film interactions modify or introduce new functionalities into the deposited epitaxial thin films. One possibility is the formation of an interface layer with different physical properties from the two components ${ }^{4}$, another is the substrate-induced modification of the thin film structure by epitaxial strain. Indeed strain engineering has become one of the most popular routes to optimize the physical properties of thin films ${ }^{5-7}$.

One of the parameters governing the physics of manganites is the geometry of the $\mathrm{MnO}_{6}$ octahedra in the perovskite structure. The strain-induced elongation, compression or rotation of the $\mathrm{MnO}_{6}$ units lead to crystal field splitting of the $\left(\mathrm{x}^{2}-\mathrm{y}^{2} / 3 \mathrm{z}^{2}-\mathrm{r}^{2}\right)$ levels, thus modifying their electron occupancy and leading to complex orbital reconstruction ${ }^{7-}$ 9. Thus manganites are good candidates among the complex oxides for the tuning of physical properties by controlling the electron occupancy of the Mn 3d orbitals via strain. For instance, it has already been extensively reported for $\mathrm{La}_{\mathrm{x}} \mathrm{Sr}_{1-\mathrm{x}} \mathrm{MnO}_{3}$ that 
tensile strain favors $\mathrm{x}^{2}-\mathrm{y}^{2}$ occupancy (the A-type antiferromagnetic (AFM) structure) and the compressive strain favors $3 z^{2}-r^{2}$ occupancy (the C-type AFM structure) ${ }^{10-13}$. As a consequence, the interface with the substrate or the surface of a ferromagnetic (FM) (conducting) manganite may have a tendency toward AFM (insulator), a phenomenon that has motivated an extensive literature. ${ }^{8,14-21}$

The work presented here is the first direct observation of the strain-induced segregation of a nonferromagnetic layer (NFL) of AFM character at the top surface of ferromagnetic epitaxial $\mathrm{La}_{2 / 3} \mathrm{Ca}_{1 / 3} \mathrm{MnO}_{3}$ (LCMO) thin films as a function of the growth conditions. This is achieved by imaging the magnetization state of the films at nanometer scale using electron holography, which is a high-resolution electron interferometric technique. This heteromagnetic structure can be in fact exchange coupled, so our findings evidence unambiguously the possibility of engineering the formation of an exchange biased FM-AFM bilayer in a single homogeneous epitaxial thin film. This opens a new route to develop fully integrated multifunctional building blocks for spintronic devices such as spin valves or magnetic tunnel junctions.

X-ray diffraction shown in Figure 1(a) evidences the structural properties of LCMO grown by pulsed laser deposition (PLD) for three different substrates: (100)-oriented $\mathrm{SrTiO}_{3}$ (STO), $\left(\mathrm{LaAlO}_{3}\right)_{0.29}-\left(\mathrm{Sr}_{0.5} \mathrm{Al}_{0.5} \mathrm{TaO}_{3}\right)_{0.71}$ (LSAT) and $\mathrm{LaAlO}_{3}$ (LAO). Strained LCMO films present an in-plane $a_{L C M O}$ well adapted to that of the substrate producing a uniform biaxial strain (see Supporting Information, SI), while different out-of-plane parameters $\left(c_{L C M O}\right)$ are found as a function of the substrate and the laser ablation frequency. The value of $c_{L C M O}$ is determined from the position of the 002 reflection peak in the diffraction patterns. The lattice mismatch, defined as $f_{\text {subs }}=100 \times\left(a_{\text {subs }}-\right.$ $\left.a_{L C M O}\right) / a_{\text {subs }}$, is used to define the strength and sign of the epitaxial strain. While LCMO grows virtually unstrained on LSAT $\left(f_{L S A T}=+0.05 \%\right)$, STO introduces a moderate tensile stress $\left(f_{\text {STO }}=+0.93 \%\right)$, with a subsequent reduction of the out-of-plane lattice parameter $c_{L C M O}$ following the Poisson's effect, and LAO induces a strong compressive strain $\left(f_{L A O}=-2.01 \%\right)$ and therefore a remarkable increase of $c_{L C M O}$. Even though $a_{L C M O}$ is fixed for each substrate, $c_{L C M O}$ can be slightly modulated by tuning the growth conditions. LCMO grown on STO upon different laser frequencies gives rise to slightly different $c_{L C M O}$, higher for a $10 \mathrm{~Hz}$ frequency than at $2 \mathrm{~Hz}$. As we will see below, these small changes produce important variations in the magnetic properties of the film. 
An interesting correlation between structure and magnetism is found by analyzing the magnetization hysteresis loops shown in Figure 1(b). While the relaxed LCMO grown on LSAT shows a high magnetization state close to bulk $\left(\mathrm{M}_{\mathrm{S}} \sim 640 \mathrm{emu} / \mathrm{cm}^{3}\right)$, the high compressive strain of LAO causes a strongly decreased saturation magnetization $(\sim 175$ $\mathrm{emu} / \mathrm{cm}^{3}$ ). Contrary to the case of STO, it is noteworthy that LCMO grown on LAO at other frequencies $(2 \mathrm{~Hz}, 20 \mathrm{~Hz}$, not shown here) also presents the magnetization reduction. Similarly to the evolution of $c_{L C M O}$, saturation magnetization of LCMO on STO depends on the laser frequency, and slow growth $(2 \mathrm{~Hz})$ produces optimum $M_{S}$ while higher frequency $(10 \mathrm{~Hz})$ reduces significantly the magnetization value at saturation to $\sim 440 \mathrm{emu} / \mathrm{cm}^{3}$.
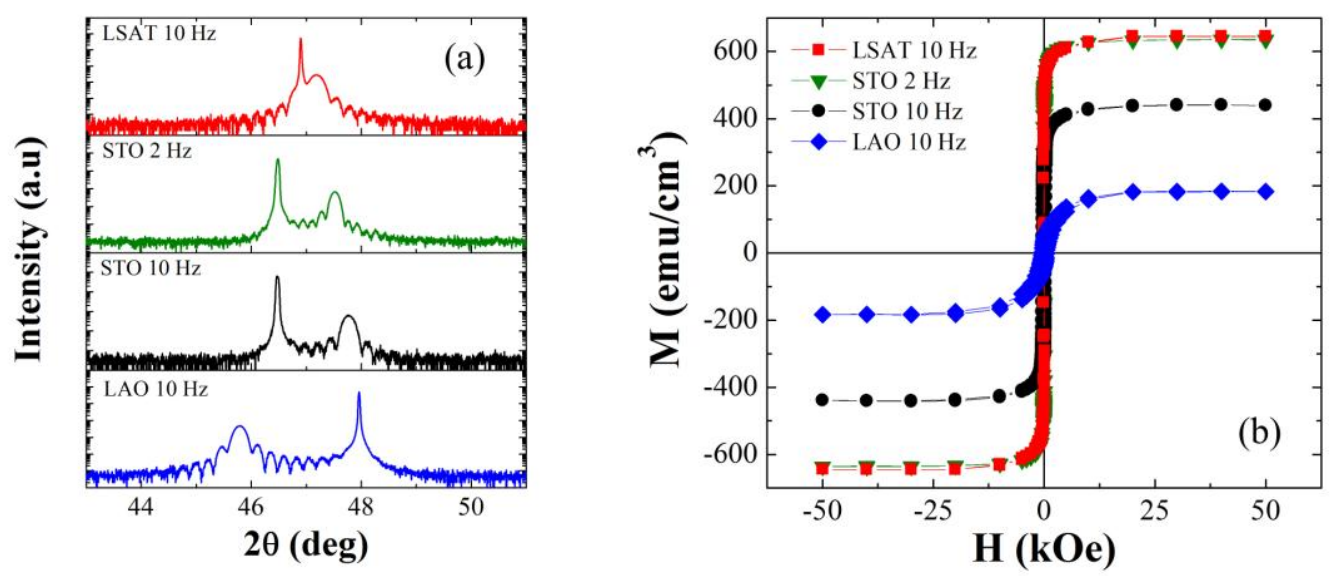

Figure 1. Macroscopic structural and magnetic characterization of the LCMO films. (a) $\theta$ $2 \theta x$-ray diffraction patterns around the (002) reflection of epitaxial LCMO thin films grown on LAO, LSAT, STO substrates, showing the different out-of-plane lattice parameter of the films with respect to the substrates used. Note that the sharp peaks represent the substrate and the rounded oscillatory reflections are originated by the films. (b) Magnetization hysteresis loops of the same LCMO thin films measured at $10 \mathrm{~K}$ showing the different values of saturation magnetization as a function of the substrate and growth conditions.

The influence of the crystal deformation on the magnetic properties of the LCMO films is more clear if we plot in Figure 2 the main magnetic parameters, saturation magnetization $\left(M_{S}\right)$ and coercivity $\left(H_{C}\right)$, as a function of tetragonality, defined as $\tau=\mid c$ $a \mid / a$ (Figure 2). The lattice parameter $a$ is always defined by the substrate, and $c$ corresponds to the out-of-plane parameter of the LCMO film. The different points correspond to samples grown on different batches. The macroscopic magnetic behavior of LCMO is governed by the tetragonality of the lattice. Saturation magnetization is kept constant (within the experimental error) and close to the bulk value for 
tetragonality values below a certain critical value $\tau_{C} \sim 0.024$. Above this value the magnetization decreases dramatically, confirming that strain-driven tetragonality is a key parameter for the growth of fully magnetized LCMO films. The case of LCMO grown on STO is particularly interesting, as it appears to lie near the crossover between a fully magnetized and ferromagnetically-depressed manganite. Fine-tuning the growth conditions, in this case the laser frequency, triggers the subtle change of tetragonality required to produce one state or another. Similar dependencies of the saturation magnetization have been observed as a function of the substrate used in half-doped LCMO and $\mathrm{LSMO}^{13}$. Interestingly, coercivity follows the same evolution, presenting very low values ( $<150 \mathrm{Oe}$ ) for low tetragonality and much higher ones above $\tau_{C}$, ranging between 400-600 Oe, which is the indication of a radical change in the magnetic state of the highly strained thin films, as shown later. 


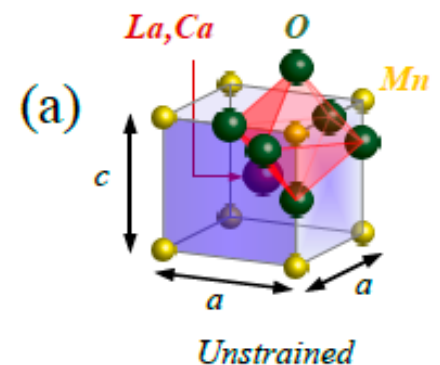

Unstrained

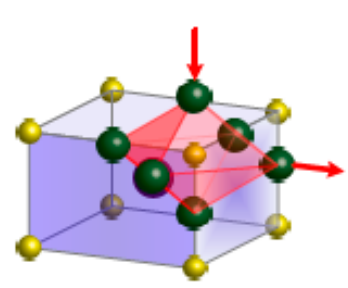

Tensile

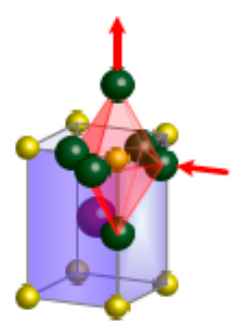

Compressive
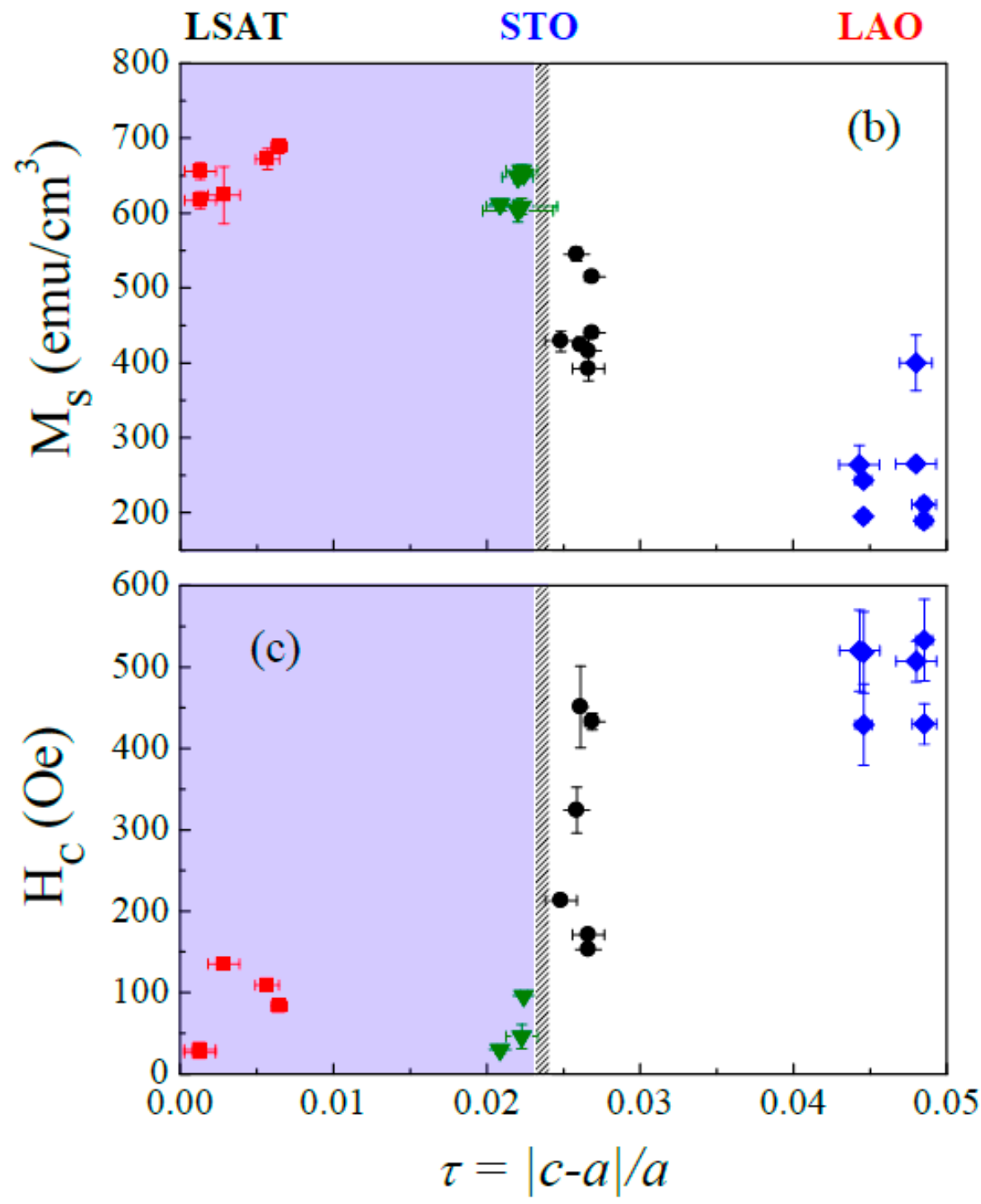

Figure 2. Tetragonality as the control parameter for suppressed ferromagnetism of strained LCMO. (a) Sketches of the type of crystal distortions are inserted in the top of the figure, where the red arrows represent the induced strain directions. (b) Saturation magnetization $\left(M_{S}\right)$ and (c) coercive field $\left(H_{C}\right)$ for different batches of LCMO films on LSAT (red squares), STO (green triangles for high magnetization, black circles for low magnetization) and LAO (blue diamonds) substrates as a function of $\tau=|c-a| / a$, where $a$ and $c$ are the in- and out-of-plane lattice parameters of the film, respectively. 
Once evidenced the influence of the structural parameters, particularly of tetragonality, in depressing the ferromagnetism of LCMO, we have carried out the direct imaging of the remanent magnetization inside the LCMO single layers by electron holography $(\mathrm{EH})$ at $100 \mathrm{~K}$ in three representative LCMO samples (see experimental details in Supporting Information). For this purpose, we have analyzed LCMO films grown on LSAT at 10 $\mathrm{Hz}$ (thickness of $43 \mathrm{~nm}$ ), on STO at $2 \mathrm{~Hz}(60 \mathrm{~nm})$, and on STO at $10 \mathrm{~Hz}(60 \mathrm{~nm})$. These results are summarized in Figures 3(a-c), respectively, showing the electron wave amplitude, the magnetic phase shift, and the magnetic flux extracted from the holograms, and line profiles extracted from the magnetic phase shift images in a direction perpendicular to the interfaces of the three samples (right columns in Fig. 3). For LCMO films with macroscopic magnetization near bulk, grown on LSAT [Fig. 3(a)] and on STO at $2 \mathrm{~Hz}$ [Fig. 3(b)], magnetic flux images show that the films are completely magnetized, and the magnetization is aligned parallel to the substrate-film interface. However, the LCMO film grown on STO at $10 \mathrm{~Hz}$ is only partially magnetized, see Fig. 3(c). As we see in the magnetic flux image, only the first $35 \mathrm{~nm}$ of the film close to substrate/film interface present a clear ferromagnetic behavior with the magnetization parallel to the substrate-film interface, with magnetic flux lines parallel to the substrate plane. In the line profile we find that while there is a strong linear variation of $\varphi_{M}$ at the first $35 \mathrm{~nm}$ of thickness, a very weak and noisy phase variation is observed at the top $24 \mathrm{~nm}$ of the layer. This indicates the appearance of a nonferromagnetic layer (NFL) at the surface of the LCMO film. The observation of such NFL on the top surface of LCMO is consistent with the saturation magnetization reduction observed macroscopically, and its correlation with tetragonality, but does not provide a definitive answer about the magnetic state of this NFL and the drastic increase of coercivity. 

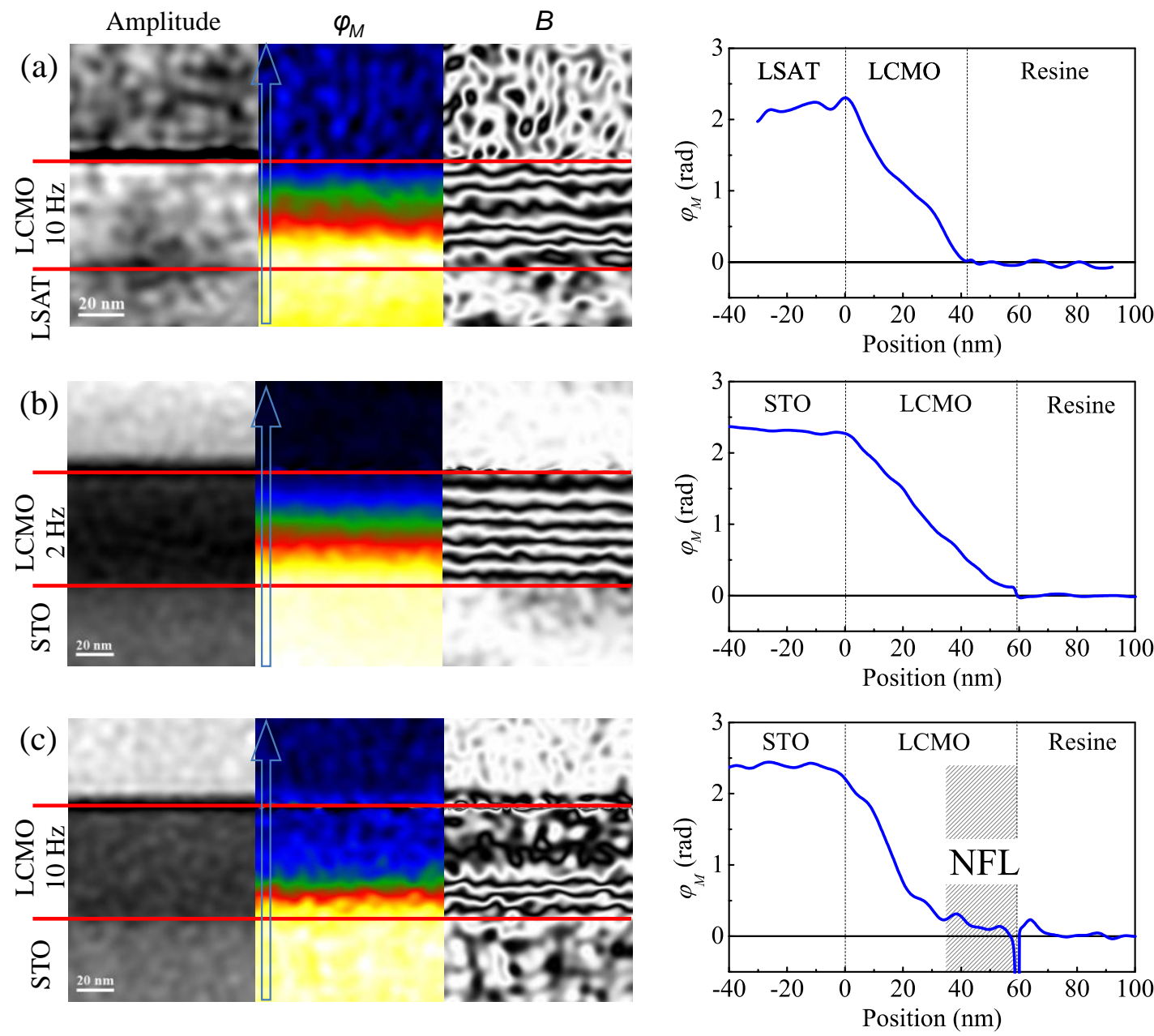

Figure 3. Imaging the magnetism of strained LCMO films by electron holography

at $100 \mathrm{~K}$. (a) Amplitude, magnetic phase shift $\left(\varphi_{M}\right)$, and magnetic flux $(\boldsymbol{B})$ shown as the cosinus of $\varphi_{M}$ for the LCMO thin films grown at different substrates (i.e., different crystal distortions): (a) LSAT, $\tau=0.005$; (b) STO (2 Hz), $\tau=0.022$; and (c) STO (10 $\mathrm{Hz}$ ), $\tau=0.025$. In the right side of (a), (b) and (c), and under (d), profiles of the magnetic phase shift images are plotted.

Previous works have demonstrated that the substrate-induced strain effect plays an important role in the formation of an NFL, commonly referred in the literature as dead layer, either close to the substrate-film interface or the free surface of the film ${ }^{17,19,22}$. The loss of ferromagnetism can have different origins, some of them of chemical nature such as oxygen vacancies that expand the lattice unit cell volume ${ }^{23}$, Mn valence instabilities at the surface of LCMO grown on LAO due to the formation of $\mathrm{Mn}^{2+}$ by air exposure $^{24}$, cation segregation ${ }^{25,26}$ or interface chemical diffusion ${ }^{27}$. A chemical origin for the NFL layer in our samples has been discarded by a careful spectroscopic analysis 
carried out by STEM-EELS on the LCMO layer grown at $10 \mathrm{~Hz}$ on STO. These studies (see Supporting Information) have revealed that the chemical composition and the nominal oxidation state of the film is homogeneous throughout the whole layer thickness at room temperature, the latter remarkably coincident with the nominal $\mathrm{Mn}$ valence of $+3.34(1)$. Thus, inhomogeneity and lack of stoichiometry (i.e. cation segregation or oxygen vacancies) are not the cause of the formation of the observed NFL. As explained before, the ferromagnetic ordering could also be suppressed by structural modifications related to the film growth modifying the magnetic ordering. Tiny perturbations caused by biaxial strain may break the degeneracy of Mn $3 \mathrm{~d} \mathrm{e}_{\mathrm{g}}$ and $t_{2 g}$ orbitals due to the tetragonal distortion ${ }^{9,28}$. This orbital reconstruction causes a selective orbital occupancy that suppresses the FM order. Furthermore, symmetry breaking at the surface lead to a suppression of double exchange interaction, which would favor an AFM ordering at the surface as demonstrated in different theoretical works $^{21,29}$

Inspired by these predictions, macroscopic magnetic measurements have been carried out to detect a possible AFM nature of the NFL. An indirect method to elucidate it is to perform hysteresis cycles at low temperature $(10 \mathrm{~K})$, after zero field cooling (ZFC) and field cooling (FC) processes in order to detect the existence of exchange bias due to an exchange coupling effect between the FM and AFM layers ${ }^{30}$. ZFC and FC hysteresis cycles for LCMO films grown on different substrates are represented in Fig. 4. For the FC hysteresis loops, the samples were cooled down at 100, 1000 and $1500 \mathrm{Oe}$, and the cycles were performed ranging the magnetic field between +50 to $-50 \mathrm{kOe}$, applying the magnetic field direction in the plane of the film. Figure 4(a) and (b) depicts the fully magnetized LCMO grown on STO at $2 \mathrm{~Hz}$ and on LSAT, both serves as reference specimens to detect the appearance of exchange bias. As expected, the ZFC and FC hysteresis loops do not show any significant difference. However, Figure 4 (c) and (d) shows the same experiments in ferromagnetically depressed LCMO grown on STO at $10 \mathrm{~Hz}$ and on LAO substrates. Here we can see that both FC hysteresis cycles present a remarkable shift toward negative fields of the hysteresis loops, $\mathrm{H}_{\mathrm{EX}}$, STO $=-35$ Oe and $\mathrm{H}_{\mathrm{EX}, \mathrm{LAO}}=-122 \mathrm{Oe}$. In the case of STO at $10 \mathrm{~Hz}$ a broadening is also observed. These behaviors correspond to the typical exchange bias effect occurring in hysteresis loops of FM-AFM bilayers that are exchange coupled after field cooling below the Néel temperature of the AFM layer. For our LCMO films this AFM layer would be the NFL 
detected by EH. Furthermore, the increase of $H_{c}$ observed agrees with the existence of a FM-AFM exchange coupling, see Figure 2(b).
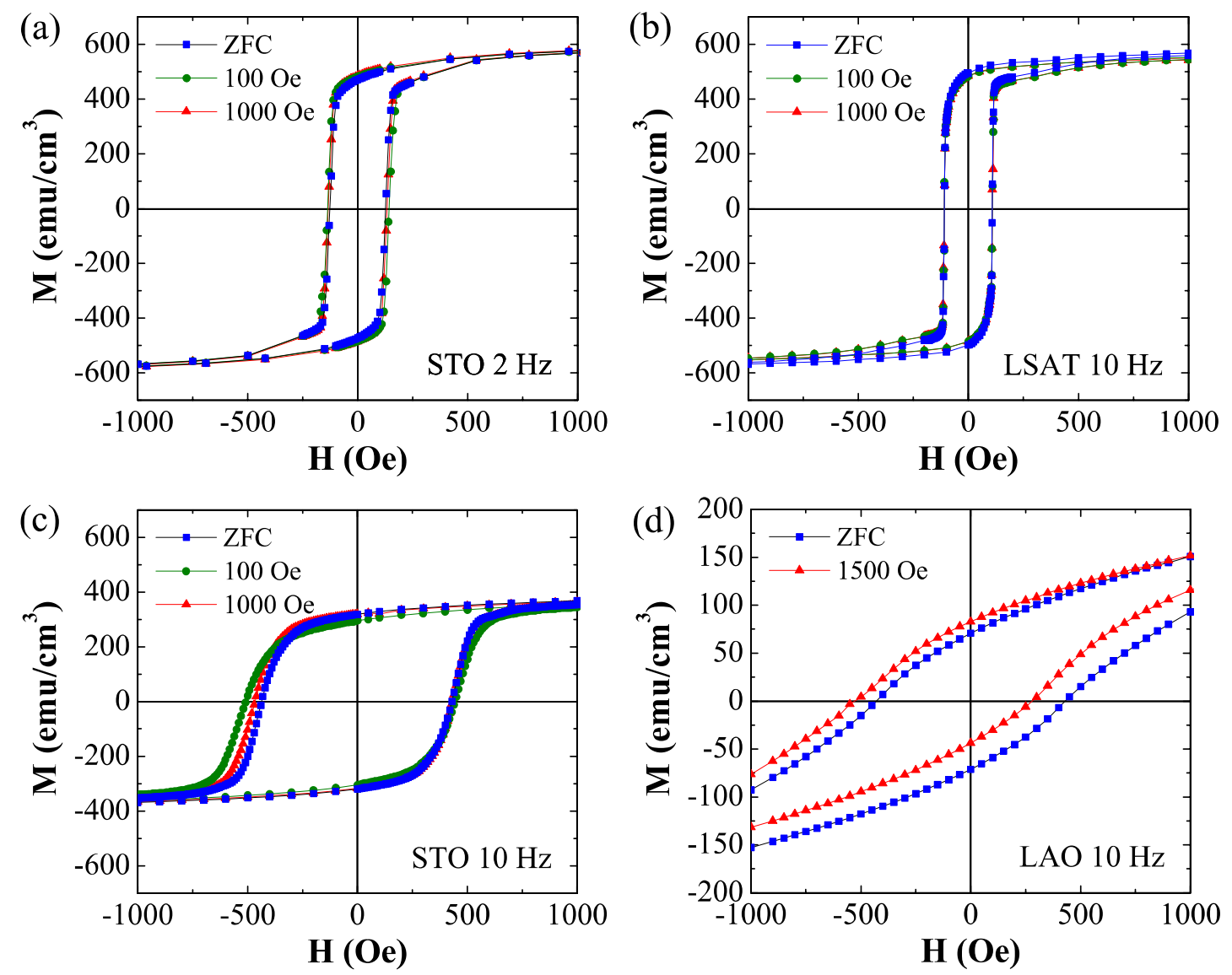

Figure 4. Evidence of the antiferromagnetic character of the top nonferromagnetic layer of LCMO. ZFC and FC magnetization hysteresis cycles at $10 \mathrm{~K}$ of LCMO grow on: (a) STO at $2 \mathrm{~Hz}$, (b) LSAT at $10 \mathrm{~Hz}$, (c) STO at $10 \mathrm{~Hz}$, and (d) LAO at $10 \mathrm{~Hz}$. The set of samples at $10 \mathrm{~Hz}$ were grown simultaneously.

The strain induced crystal distortion itself can be the physical mechanism that explains the segregation of a top AFM layer. This deformation will induce tilts and distortions of the $\mathrm{MnO}_{6}$ octahedral, modifying the $\mathrm{Mn}-\mathrm{O}$ bond distance and the angles of the Mn-OMn bonds ${ }^{20}$. This structural modifications cause radical changes in the magnetic and transport properties in manganites. Theoretical and experimental results predict that a coupling between the orbital ordering of the $e_{g}$ states and the Jahn-Teller distortions of the $\mathrm{MnO}_{6}$ octahedron lead to a magnetic transition from FM state in unstrained films to C-type antiferromagnetic state (C-AFM) at large compressive strains or A-type antiferromagnetic state (A-AFM) under large tensile strains ${ }^{9}$. Another possible phase is 
orbital glass insulator ${ }^{7,31}$, insomuch as the exchange bias effect indicates a coupling between phases with different spin orders and different interactions could be considered $^{32}$.
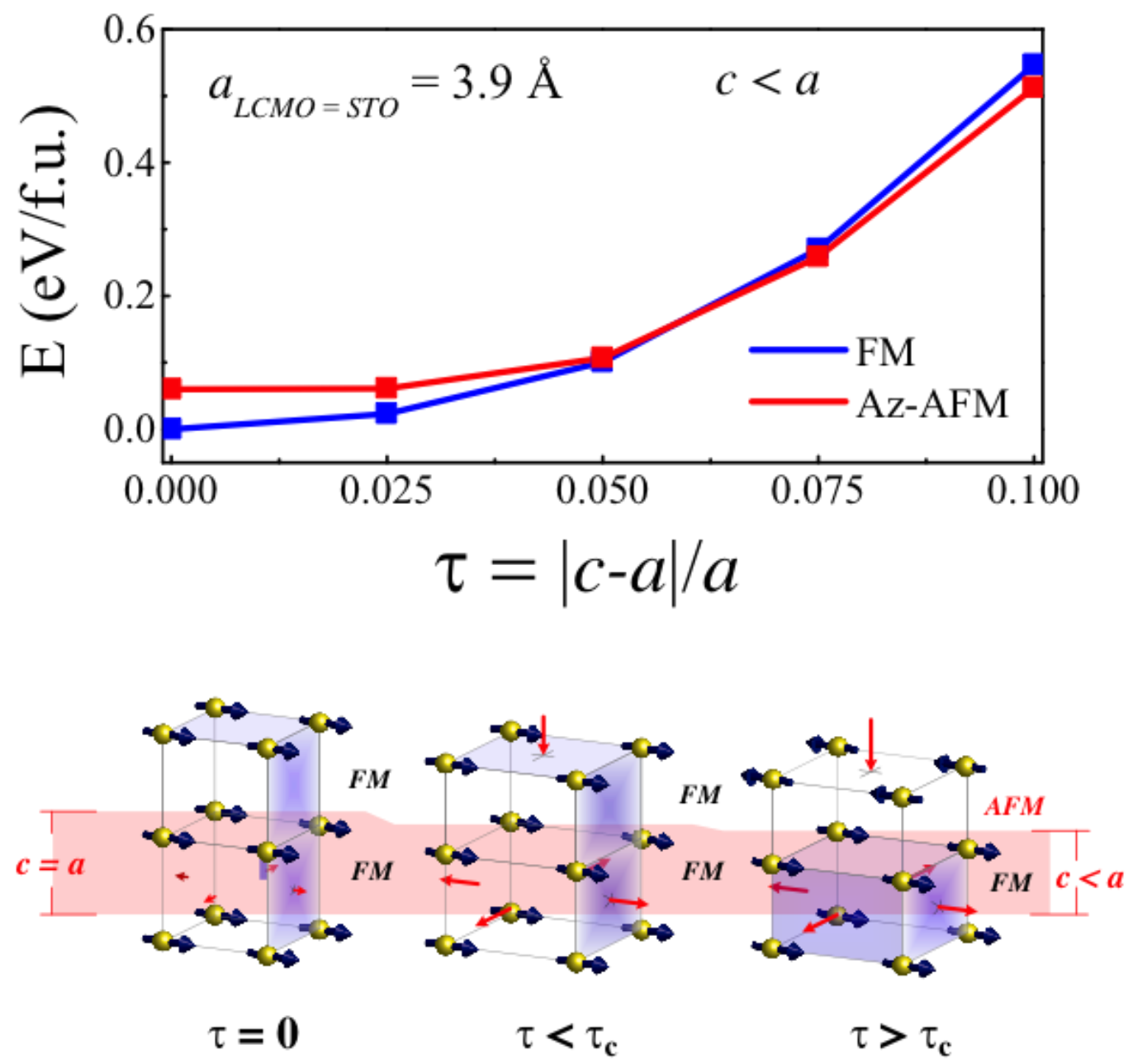

Figure 5. First-principles calculations of the magnetic ordering of strained LCMO. (a) Energy variation of the FM and $\mathrm{A}_{\mathrm{z}}-\mathrm{AFM}$ orders in LCMO as a function of the tetragonality for an in-plane lattice parameter fixed by the STO substrate $\left(a_{S T O}=3.9 \AA\right)$. (b) Schematic representation of the different magnetic orderings.

To get a deeper insight on the competition between the FM and AFM orders in strained LCMO films, we have performed DFT $+U$ calculations of LCMO structures with an inplane lattice parameter determined by STO $(\mathrm{a}=3.9 \AA)$ and LAO substrates $(\mathrm{a}=3.8 \AA$, see Supporting Information) as a function of the out-of-plane lattice parameter for 4 magnetic orderings as proposed by Colizzi et al. in $\mathrm{LSMO}^{12}$. According to Fig. 5 , for $\tau=$ 0 the FM ordering is always the most stable, independently of the in-plane lattice parameter. For $a=3.9 \AA$, the c axis is reduced by the in-plane tensile strain and our calculations predict a magnetic transition from a FM to an $\mathrm{A}_{\mathrm{z}}$-AFM ordering for $\tau>$ 
0.06. Between $\tau=0$ and $\tau=0.025$, the energy of both FM and $\mathrm{A}_{\mathrm{z}}$-AFM states increases, but the energy difference between the two orderings is reduced by approximately $40 \%$. For $\tau=0.025$, this energy difference is then $37.9 \mathrm{meV} / \mathrm{f} . \mathrm{u}$, and only $6.3 \mathrm{meV} / \mathrm{f} . \mathrm{u}$ if $\tau$ increases up to 0.05 . Thus, such small energy difference suggests that a coexistence of the two orderings is possible considering the thermal energy and additional local perturbations not taken into account in the present calculations. Furthermore, our calculations model the LCMO ground state at $0 \mathrm{~K}$ and does not contemplate any possible temperature dependence of the crystal structure (i.e. the tetragonality). However magnetic transitions in manganites are often coupled with strong lattice distortions ${ }^{33,34}$. Thus the possibility of a low temperature structurally inhomogeneous film, particularly with two regions with remarkably different tetragonality, coupled with the magnetic ordering is another ingredient that should be considered. As already discussed, these perturbations can result from the presence of a free surface and an interface with the substrate, or small deviations from the nominal stoichiometry undetected by our experimental techniques. Eventually it becomes clearer for $\tau \sim 0.06$, where a crossover takes place after which the AFM ordering is favored energetically.

Our findings evidence both experimentally and theoretically that the strain engineering of a LCMO film structurally and chemically homogeneous at room temperature can produce two spatially segregated layers with different magnetic orderings. In addition, symmetry breaking contributes to this effect by the relaxation of the long-range interactions at the surface. Our approach has the great advantage that the two functional phases are inherently coupled through a single flat and smooth interface to provide a combined functionality that was absent in the original unstrained material. As we have demonstrated, the ferromagnetic layer can be spontaneously pinned by exchange bias without the use of a dedicated AFM pinning layer made of another material. This new route could be exploited in the field of spintronic devices for the fabrication of more simple spin valves or magnetic tunnel junctions with other complex oxide thin films on the verge of phase coexistence regimes in which epitaxial strain may be able to stabilize a FM-AFM phase segregation. Eventually innumerable applications could be envisaged given that following the same strategy different transport (metal, semiconductor, and insulator) or ferroic (ferromagnetic, ferroelectric, and ferroelastic) properties can be conferred to the phase segregated states.

\section{REFERENCES}


1. Cheong, S.-W.; Mostovoy, M. Nat Mater 6, 13-20 (2007).

2. Llordés, A.; Palau, A.; Gázquez, J.; Coll, M;. Vlad, R.; Pomar, A.; Arbiol, J.; Guzmán, R.; Ye, S.; Rouco, V.; Sandiumenge, F.; Ricart, S.; Puig, T.; Varela, M.; Chateigner, D.; Vanacken, J.; Gutiérrez, J.; Moshchalkov, V.; Deutscher, G.; Magen, C.; Obradors, X. Nat. Mater. 2012, 11, 329-336.

3. Habermeier, H.-U. Mater. Today 2007, 10, 34.

4. Hwang, H. Y.; Iwasa, Y.; Kawasaki, M.; Keimer, B.; Nagaosa, N.; Tokura, Y. Nat. Mater. 2012, 11, 103.

5. Bozovic, I.; Logvenov, G.; Belca, I.; Narimbetov, B.; Sveklo, I. Phys. Rev. Lett. 2002 , 89, 107001.

6. Haeni, J. H.; Irvin, P.; Chang, W.; Uecker, R.; Reiche, P.; Li, Y.L.; Choudhury, S.; Tian, W.; Hawley, M.E.; Craigo, B.; Tagantsev, A.K.; Pan, X.Q.; Streiffer, S.K. Chen; L.Q.; Kirchoefer, S.W.; Levy, J.; Schlom, D.G. Nature 2004, 430, 758.

7. Abad, L.; Laukhin, V.; Valencia, S.; Gaup, A.; Gudat, W.; Balcells, L.; Martínez, B. Adv. Funct. Mater. 2007, 17, 3918.

8. Tebano, A.; Aruta, C.; Sanna, S.; Medaglia, P.G.; Balestrino, G.; Sidorenko, A.A.; De Renzi, R.; Ghiringhelli, G.; Braicovich, L.; Bisogni, V.; Brookes, N.B. Phys. Rev. Lett. 2008, 100, 137401 .

9. Tokura, Y.; Nagaosa, N. Science 2000, 288, 462.

10. Konishi, Y.; Fang, Z.; Izumi, M.; Manako, T.; Kasai, M.; Kuwahara, H.; Kawasaki, M.; Terakura, K.; Tokura, Y. J. Phys. Soc. Japan 1999, 68, 3790.

11. Fang, Z.; Solovyev, I. V; Terakura, K. Phys. Rev. Lett. 2000, 84, 3169.

12. Colizzi, G.; Filippetti, A.; Cossu, F.; Fiorentini, V. Phys. Rev. B 2008, 78, 235122.

13. Gutiérrez, D.; Radaelli, G.; Sánchez, F.; Bertacco, R.; Fontcuberta, J. Phys. Rev. B 2014, $89,75107$.

14. Sandiumenge, F.; Santiso, J.; Balcells, Ll.; Konstantinovic, Z.; Roqueta, J.; Pomar, A.; Espinós, J.P.; Martínez, B. Phys. Rev. Lett. 2013, 110, 107206.

15. Yamada, H.; Ogawa, Y.; Ishii, Y.; Sato, H.; Kawasaki, M.; Akoh, H.; Tokura, Y. Science 2004, 305, 646.

16. Infante, I. C.; Sánchez, F.; Fontcuberta, J.; Wojcik, M.; Jedryka, E.; Estradé, S.; Peiró, F.; Arbiol, J.; Laukhin, V.; Espinós J.P. Phys. Rev. B 2007, 76, 224415.

17. Freeland, J. W.; Kavich, J.J.; Gray, K.E.; Ozyuzer, L.; Zheng, H.; Mitchell, J.F.; Warusawithana, M.P.; Ryan, P.; Zhai, X.; Kodama, R.K.; Eckstein, J.N. J. Phys. Condens. Matter 2007, 19, 315210.

18. Huijben, M.; Martin, L.W.; Chu, Y.-H.; Holcomb, M.B.; Yu, P.; Rijnders, G.; Blank, D. H. A.; Ramesh, R. Phys. Rev. B 2008, 78, 94413. 
19. Pesquera, D.; Herranz, G.; Barla, A.; Pellegrin, E.; Bondino, F.; Magnano, E.; Sanchez, F.; Fontcuberta, J. Nat. Commun. 2012, 3, 1189.

20. He, J.; Borisevich, A.; Kalinin, S. V.; Pennycook, S. J.; Pantelides, S. T. Phys. Rev. Lett. 2010, 105, 227203.

21. Calderón, M. J.; Brey, L; Guinea, F. Phys. Rev. B 199, 60, 6698.

22. Valencia, S.; Peña, L.; Konstantinovic, Z.; Balcells, L1.; Galceran, R.; Schmitz, D.; Sandiumenge, F.; Casanove, M.; Martínez, B. J. Phys. Condens. Matter 26, 166001 (2014).

23. Schumacher, D.; Steffen, A.; Voigt, J.; Schubert, J.; Brückel, Th.; Ambaye, H.; Lauter, V. Phys. Rev. B 2003, 88, 144427.

24. Valencia, S.; Gaupp, A.; Gudat, W.; Abad, Ll.; Balcells, L1.; Cavallaro, A.; Martínez, B.; Palomares, F.J. Phys. Rev. B 2006, 73, 104402.

25. Estradé, S.; Rebled, J.M.; Arbiol, J.; Peiró, F.; Infante, I.C.; Herranz, G.; Sánchez, F.; Fontcuberta, J.; Córdoba, R.; Mendis, B.G.; Bleloch, A.L. Appl. Phys. Lett. 2009, 95, 072507.

26. Song, J. H.; Susaki, T.; Hwang, H. Y. Adv. Mater. 2008, 20, 2528.

27. Kourkoutis, L. F.; Song, J. H.; Hwang, H. Y.; Muller, D. A. Proc. Natl. Acad. Sci. U. S. A. 2010, 107, 11682.

28. Fang, Z.; Solovyev, I; Terakura, K. Phys. Rev. Lett. 2000, 84, 3169.

29. Cossu, F.; Schwingenschlögl, U.; Colizzi, G.; Filippetti, A.; Fiorentini, V. Phys. Rev. B 2013, 87, 214420.

30. Nogués, J.; Sort, J.; Langlais, V.; Skumryev, V.; Suriñach, S.; Muñoz, J.S.; Baró M. D. Phys. Rep. 2005, 422, 65.

31. Cui, B.; Song, C.; Wang, G. Y.; Mao, H. J.; Feng, F.; Pan, F. Sci. Rep. 2013, 3, 2542.

32. Kiwi, M. J. Magn. Magn. Mater. 2001 234, 584.

\section{ACKNOWLEDGEMENTS}

This work was supported by Spanish Ministry of Science through projects No. MAT2011-28532-C03-02 and MAT2011-27553-C02 including FEDER funding, by the Aragón Regional Government through projects E26 and CTPP4/11, by the Universidad de Zaragoza through the contract PIF-UZ-2009-CIE-02, and by the European Union under the Seventh Framework Programme under a contract for an Integrated Infrastructure Initiative Reference 312483-ESTEEM2. It was granted access to the HPC resources of CALMIP under the allocations P1313 (2013-2014) and GENCI-CINES 
(Grant 2013- and 2014-c2014096949). Experimental support for lamellae preparation by Laura Casado from LMA (University of Zaragoza) is acknowledged. 